\title{
Manipulating the Structure of Ion Coulomb Crystals with Light
}

\author{
P. Horak ${ }^{l}$, A. Dantan ${ }^{2}$, M. Drewsen ${ }^{2}$ \\ 1. Optoelectronics Research Centre, University of Southampton, Southampton SO17 1DP, United Kingdom \\ 2. QUANTOP, Danish National Research Foundation Center for Quantum Optics, Department of Physics and Astronomy, University of \\ Aarhus, DK-8000 Aarhus C., Denmark
}

Coulomb crystallisation of laser-cooled ensembles of ions in harmonic traps has been studied experimentally with many spectacular results in recent years [1-3]. It is well known that for sufficiently large numbers of ions the ground state of a Coulomb crystal in such a system has a body-centred cubic (bcc) structure. However, in experiments with crystals of up to a few $10^{4}$ ions, structural transitions between bcc and, for example, facecentred cubic (fcc) or hexagonal close packed (hcp) structures are observed [4]. For constant ion densities, the lattice spacing of the planes of highest density differs only by about $3 \%$ between bec and fcc structures and simulations predict very small energy differences, thus thermal effects even at temperatures in the mK regime are sufficient to excite these transitions. Here we study numerically the possibility of controlling and switching the crystal structure with the help of periodic potentials generated by the dipole forces of standing-wave light fields.

Our model is based on semiclassical Monte-Carlo simulations of $N$ two-level ions including Coulomb forces, a harmonic trap (frequencies $f_{z}, f_{r}$ ), 3D Doppler cooling, a tunable sinusoidal potential (periodicity $\Lambda$, depth $V_{0}$ ), and momentum diffusion induced by photon scattering. Simulations are initialised with zero-temperature ion crystals where a fraction of "core" ions have been fixed to a given crystal structure (fcc or bcc), and the remaining ions have been relaxed to form a minimum energy shell around this crystalline core. During the subsequent time evolution we fit every one of the core ions and its nearest neighbours with bcc and fcc/hcp structures. The root-mean-square (rms) deviations of these fits averaged over 100 realisations are plotted in Fig.1(a) for initial fcc and bcc structures without a periodic potential. We see that the bcc crystal largely maintains its structure, while the fcc crystal quickly deteriorates to a state where fcc and bcc fits exhibit similar rms. The inset shows a particular single simulation where the crystal undergoes a complete transition from fcc to bcc in two steps, which we interpret as the re-arrangement of subdomains in the crystal structure.

Fig.1(b) demonstrates the stabilising effect of a standing-wave dipole potential. The inset shows how various standing-wave periodicities favour one of the crystal structures over the other. Here we choose $\Lambda$ to be compatible with an fcc structure with (110) planes orthogonal to the trap Z-axis. Relatively deep optical potentials of the order of a few $\mathrm{mK}$ are required to stabilise the crystal in the fcc state with a fidelity similar to a bcc crystal without a potential (indicated by the dashed line). This is in agreement with our earlier observation that structural transitions occur first in small subdomains with correspondingly large thermal fluctuations.

We will further analyse the energy scales involved with crystal transitions, defects, finite size, and surface effects. Finally, we will discuss the possibility of switching between crystal structures by changing the standingwave potential during the dynamical evolution of the ion crystal.
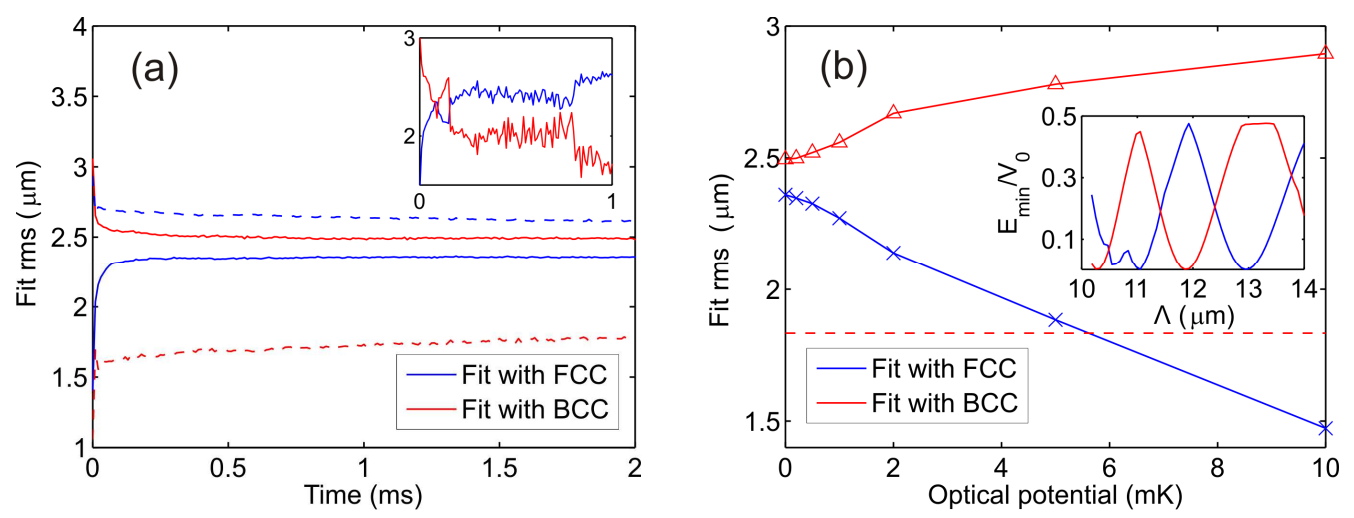

Fig. 1. (a) Time evolution of the rms of fits with fcc and bcc structures for initially cold fcc (solid) and bcc (dashed) crystals without external potential. Inset: Sample trajectory exhibiting a change of crystal structure. (b) Structural fits after $10 \mathrm{~ms}$ of propagation from initially cold fcc crystals versus depth of an added standing wave potential with $\Lambda=13 \mu \mathrm{m}$ (equal to the lattice spacing in the fcc structure). Inset: Minimum potential energy of bcc and fcc crystals in a standing wave potential versus $\Lambda$. Simulations are for 1000 ions, crystal structure fits are to the core 50 ions only. The Doppler temperature is $0.5 \mathrm{mK}$, trap frequencies are $f_{z}=f_{r} / 2=100 \mathrm{kHz}$.

\section{References}

[1] P.F. Herskind et al., "Realization of collective strong coupling with Coulomb crystals in an optical cavity", Nature Phys. 5, 494 (2009).

[2] M.J. Biercuk et al.,"Optimized dynamical decoupling in a model quantum memory", Nature 458, 996 (2009).

[3] P.F. Staanum et al., "Rotational laser cooling of vibrationally and translationally cold molecular ions", Nature Phys. 6, 271 (2010).

[4] A. Mortensen et al., "Observation of 3D long-range order in small ion Coulomb crystals in an rf trap", Phys. Rev. Lett. 96, 103001(2006). 